\title{
Influencia de la consulta de enfermedad renal crónica avanzada en la elección de modalidad de terapia renal sustitutiva
}

\author{
Carmen Caro Domínguez, Luis Garrido Pérez, María Sanz Turrado \\ Facultad de Medicina y Enfermería de Córdoba. España
}

\section{Resumen}

Introducción: La enfermedad renal crónica constituye un problema de salud pública por su elevada incidencia y prevalencia, importante morbimortalidad y coste asistencial. Un aspecto fundamental para el paciente es la elección de modalidad de terapia sustitutiva renal. En este sentido, la consulta de enfermedad renal crónica avanzada o prediálisis, puede jugar un papel fundamental.

Objetivo: Conocer producción científica sobre la influencia de la consulta de enfermedad renal crónica avanzada en la elección de modalidad de diálisis por parte del paciente.

Metodología: Revisión bibliográfica para la que se realizaron búsquedas en las bases de datos de PubMed, Scielo, Science Direct, Proquest y Google Académico. Se analizaron los artículos que trataban la consulta prediálisis, variables que influyeran en la elección de modalidad de diálisis y satisfacción del paciente.

Resultados: Se han revisado 25 artículos publicados en los años 2002-2014, de diseño observacional descriptivo y de cohortes. Se ha encontrado relación en la elección de las técnicas domiciliarias con la existencia de un programa de educación prediálisis, la información que ofrece enfermería, la entrada programada en diálisis, menor edad, menor comorbilidad y factores socioeconómicos o estructurales.

Conclusion: Los factores que favorecen la elección de las técnicas de diálisis domiciliarias son la existencia de consulta de enfermedad renal crónica avanzada y la

Correspondencia:
Carmen Caro Domínguez
Facultad de Medicina y Enfermería
Avda. Menéndez Pidal s/n. 14004 Córdoba. España
E-mail: n22cadoc@uco.es

referencia oportuna del paciente a dicha consulta, ser joven, menor comorbilidad y la necesidad de contención de costes. Esta elección se ve perjudicada por factores estructurales. Las terapias domiciliarias producen mayor satisfacción en los pacientes.

PALABRAS CLAVE

- EDUCACIÓN PREDIÁLISIS

- TERAPIA RENAL SUSTITUTIVA

- SELECCIÓN DEL PACIENTE

- SATISFACCIÓN DEL PACIENTE

Influence of the consultation of advanced chronic kidney disease in the choice of renal replacement therapy modality

\section{Abstract}

Introduction: Chronic kidney disease is a public health problem due to its high incidence and prevalence, important morbidity and mortality, and cost of care. A fundamental aspect for the patient is the choice of modality of renal replacement therapy. In this sense, the consultation of advanced chronic renal disease or predialysis, can play a fundamental role.

Objective: To know scientific production on the influence of the consultation of advanced chronic renal disease in the choice of renal replacement therapy modality from the patient perspective.

Methodology: Bibliographic review for which the databases of PubMed, Scielo, Science Direct, Proquest and Google Scholar were searched. We analyzed those articles that addressed the pre-dialysis consultation, variables that influenced the choice of dialysis modality and patient satisfaction. 
Results: We have reviewed 25 articles published in the years 2002-2014, both descriptive observational and cohort design. We have found a relationship in the choice of domiciliary techniques with the existence of a predialysis education program, the information offered by nursing, the programmed entrance into dialysis, younger age, lower comorbidity and socioeconomic or structural factors.

Conclusion: The factors facilitating the choice of home dialysis techniques are the existence of advanced chronic kidney disease consultation and the patient's timely reference to such consultation, being young, reduced comorbidity and the need for cost containment. This choice is hampered by structural factors. Home therapies produce greater patient satisfaction.

\section{KEYWORDS}

- PREDIALYSIS EDUCATION

- RENAL REPLACEMENT THERAPY

- PATIENT SELECTION

- PATIENT SATISFACTION

\section{Introducción}

La enfermedad renal crónica es un importante problema de salud pública por su elevada incidencia, prevalencia, importante morbimortalidad y coste asistencial ${ }^{1}$. Se define como la disminución de la función renal, expresada por un filtrado glomerular $(F G)<60 \mathrm{ml} / \mathrm{min} / 1,73 \mathrm{~m}^{2} 0$ la presencia de lesión renal persistente durante al menos tres meses según la guía KDIGO² publicada en 2013. La enfermedad renal crónica avanzada (ERCA) incluye los estadios 4 y 5 , definiéndose como la enfermedad renal crónica que cursa con $\mathrm{FG}<30 \mathrm{ml} / \mathrm{min}^{3}$.

Según los resultados del estudio EPIRCE (Epidemiología de la Insuficiencia Renal Crónica en España) promovido por la Sociedad Española de Nefrología (SEN) con el apoyo del Ministerio de Sanidad y Consumo, el 9,24\% de la población adulta sufre algún grado de enfermedad renal crónica, estando el 6,83\% en estadios 3-5, aunque existían diferencias importantes con la edad (aumento de la prevalencia en la población mayor de 64 años), haciéndose subsidiarios de tratamiento renal sustitutivo (TRS) y llegando a consumir el 2,5\% del presupuesto sanitario del país y más del $4 \%$ del presupuesto de Atención Especializada 4 . Por todo ello, es necesario enfocar el tratamiento al mayor beneficio para el paciente y también a la mejor adecuación coste-beneficio del mismo ${ }^{5}$.
Dentro de las TRS, los dos procedimientos de diálisis utilizados universalmente son la hemodiálisis (HD) y la diálisis peritoneal (DP). La HD, aunque se puede realizar en el domicilio del paciente, se lleva a cabo en centros sanitarios, tanto hospitalarios como periféricos. La DP por el contrario, es una técnica domiciliaria, realizada por el propio paciente y que ofrece dos modalidades u opciones: la diálisis peritoneal continua ambulatoria (DPCA), en la cual, el paciente se realiza una serie de intercambios manualmente durante el día, y la diálisis peritoneal automatizada (DPA), en la que, el intercambio del líquido de diálisis lo realiza una máquina de pequeño tamaño, programada para drenar e infundir el líquido a la cavidad abdominal un número determinado de veces, según las necesidades del paciente. Los intercambios en DPA se suelen realizar durante la noche mientras el paciente está dormido, dejando un volumen de líquido sin drenar en el último intercambio que permanece durante el día ${ }^{6}$.

Para que el paciente tenga la opción de elegir el tipo de procedimiento dialítico es necesaria la consulta de ERCA, donde un equipo multidisciplinar lleva a cabo el seguimiento y control de la función renal para retrasar su progresión, prevenir complicaciones, adecuar la preparación del TRS e iniciarlo en el momento adecuado de forma programada. Además, se lleva a cabo un programa de educación sanitaria por parte de enfermería, en el que se informa de la dieta, procediendo de diálisis, accesos vasculares, posibles problemas que pueden ocurrir, turnos y horarios, transporte... haciendo que, la elección de técnica de diálisis sea más libre ${ }^{7}$.

La implantación de las consultas de ERCA con este abordaje de cuidado integral es relativamente reciente con unos objetivos diferentes a la clásica consulta médica. El objetivo principal de esta consulta es proporcionar un apoyo integral al paciente con ERCA, en el que se contempla asistencia clínica, información, educación sanitaria e investigación consensuada ${ }^{8}$. Para que la información sea lo más objetiva posible se debe ofrecer de forma paulatina y reglada a medida que el paciente va asumiendo su proceso $0^{9}$; que lo ayude, junto a su familia, a tomar una decisión informada respecto a la modalidad de TRS que puede elegir, un derecho que en nuestro país está regulado por la Ley 41/2002 de 14 de noviembre ${ }^{10}$, básica reguladora de la autonomía del paciente y de derechos y obligaciones en materia de información y documentación clínica.

No obstante, no podemos simplificar la elección de TRS a que exista o no consulta ERCA, pues en esta elección influyen otros muchos factores: epidemiológi- 
cos y estructurales, condicionantes médicos, realidad socio-cultural de la población, que hace que, la elección del paciente se vea comprometida ${ }^{11}$. Pese a todo, se aconseja la remisión precoz del paciente a las unidades de nefrología, para que aumenten así las opciones reales de elección de modalidad terapéutica y disponer del tiempo suficiente de preparación en la elección del TRS ${ }^{12}$.

Por todo ello, nos planteamos el objetivo principal de conocer la bibliografía existente sobre la influencia que tiene la consulta ERCA en la elección que hace el paciente de modalidad de TRS, con los siguientes objetivos específicos:

- Determinar las principales variables que influyen en la elección de la modalidad de TRS.

- Conocer la relación entre la modalidad de TRS y grado de satisfacción con la misma.

\section{Metodología}

El diseño empleado para el estudio fue el de una revisión bibliográfica. Se realizó diversas búsquedas en varias bases de datos con el objetivo de obtener la mayor información posible sobre el tema del estudio.

Las bases de datos consultadas fueron Pubmed, Scielo, Google Académico, Proquest, Science Direct. El periodo de consulta tuvo lugar desde el 22 de enero de 2016 hasta el 18 de marzo de 2016.

El método de búsqueda consistió en utilizar palabras clave en las bases de datos, como educación prediálisis (predialysis education), terapia renal sustitutiva (renal replacement therapy), selección del paciente (patient selection), satisfacción del paciente (patient satisfaction) unidas por los operadores booleanos "and" y "or", según conviniera una u otra combinación, y empleando los distintos descriptores en singular y que estuvieran presentes en título, resumen y, si la base de datos lo permitía en las palabras clave. Además, la búsqueda se restringió a aquellos artículos que disponían de texto completo.

Los criterios de inclusión fueron:

- Que fuesen artículos originales completos o de revisión.

- Que los artículos trataran de la consulta ERCA y su función en el abordaje de los pacientes que van a iniciar TRS.
- Trabajos relacionados con los factores que condicionan la elección de la TRS.

Los criterios de exclusión fueron:

- Trabajos que no publicasen los resultados.

- Artículos cuyos resultados no respondieran a ninguno de los objetivos propuestos en esta revisión.

Una vez introducida en la base de datos los criterios de búsqueda y obtenidos los artículos correspondientes, se procedió a la lectura de título y resumen y, aquellos que no seguían los criterios de inclusión/exclusión preestablecidos, no tuvieran relación con el tema o estuvieran repetidos, se iban descartando. Los que sí los cumplían, se fueron guardando para la lectura del texto completo y después decidir si se incluían o no en el trabajo.

\section{Resultados y discusión}

En función de la base de datos consultada, los criterios de búsqueda y resultados obtenidos variaron. En la tabla 1 se puede observar el número de documentos encontrados en cada base de datos, así como el total obtenido.

\begin{tabular}{|c|c|c|c|c|c|c|}
\hline BASE DE DATOS & PUBMED & SCIELO & $\begin{array}{l}\text { SCIENCE } \\
\text { DIRECT }\end{array}$ & $\begin{array}{c}\text { GOOGLE } \\
\text { ACADÉMICO }\end{array}$ & PROQUEST & TOTAL \\
\hline $\begin{array}{l}\text { Publicaciones } \\
\text { encontradas }\end{array}$ & 17 & 20 & 11 & 32 & 13 & 93 \\
\hline $\begin{array}{l}\text { Publicaciones } \\
\text { preseleccionadas }\end{array}$ & 13 & 16 & 6 & 29 & 7 & 71 \\
\hline
\end{tabular}

De los 71 documentos preseleccionados, seleccionamos 43 artículos de acuerdo a la estrategia de búsqueda que se muestra en la Figura 1.

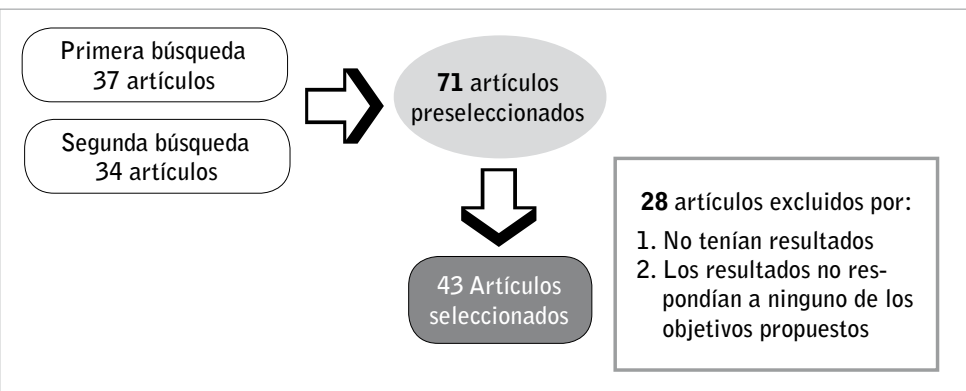

Figura 1. Número de Artículos encontrados en cada búsqueda. 


\section{[ Carmen Caro Domínguez, et al ]}

Influencia de la consulta de enfermedad renal crónica avanzada en la elección de modalidad de terapia renal sustitutiva

Tras hacer una lectura completa, se excluyeron de nuevo otros estudios que no encajaban del todo en los objetivos, quedando al final 25 artículos que son los que aparecen incluidos en la tabla 2.

Tabla 2. Clasificación de todos los artículos seleccionados para el trabajo.

\begin{tabular}{|c|c|c|c|}
\hline Autor, año, país & Muestra & Diseño del estudio & Resultados \\
\hline $\begin{array}{l}\text { Ribitsch W, et al.2013. } \\
\text { Austria }^{13}\end{array}$ & $227^{*}$ & $\begin{array}{l}\text { Estudio de cohortes } \\
\text { retrospectivo }\end{array}$ & $\begin{array}{l}\text { - Un programa de educación prediálisis tiene un } \\
\text { gran impacto en la distribución de modalidades } \\
\text { de diálisis, fomenta la referencia oportuna y } \\
\text { proporciona un enfoque de atención integral. } \\
\text { - Menor frecuencia de DP en mayores. }\end{array}$ \\
\hline $\begin{array}{l}\text { Pastor JL, Julián JC. } 2010 . \\
\text { España }^{14}\end{array}$ & $202 *$ & $\begin{array}{l}\text { Estudio observacional } \\
\text { descriptivo transversal }\end{array}$ & $\begin{array}{l}\text { - La mayor parte de los pacientes no disponen de } \\
\text { conocimiento suficiente sobre DP. } \\
\text { - A mayor edad del paciente, la información sobre } \\
\text { TRS presenta un descenso progresivo. } \\
\text { - La DP se explica a los pacientes a los que se les } \\
\text { va a prescribir y un proceso educacional influye } \\
\text { en la modalidad de diálisis elegida. }\end{array}$ \\
\hline $\begin{array}{l}\text { Goovaerts T, Jadoul M, Goffin } \\
\text { E. 2005. Bélgica }{ }^{15}\end{array}$ & $185^{*}$ & $\begin{array}{l}\text { Estudio observacional } \\
\text { retrospectivo }\end{array}$ & $\begin{array}{l}\text {-Un alto porcentaje de pacientes asistentes a un } \\
\text { programa estructurado eligenTRS de autocuidado. } \\
\text { - El número de pacientes en diálisis se hace cada } \\
\text { vez mayor y es necesario restringir recursos. }\end{array}$ \\
\hline $\begin{array}{l}\text { Ponz E, Martínez JC, } \\
\text { Marquina C et al. } 2010 . \\
\text { España }^{16}\end{array}$ & $44^{*}$ & $\begin{array}{l}\text { Estudio observacional } \\
\text { prospectivo }\end{array}$ & $\begin{array}{l}\text { - Ni los síntomas depresivos ni los rasgos de } \\
\text { personalidad influyeron en el TSR inicialmente } \\
\text { elegido, aunque pueden ser factores implicados en } \\
\text { los cambios de decisión posteriores. }\end{array}$ \\
\hline $\begin{array}{l}\text { Álvarez R, Velasco S. } 2007 . \\
\text { España }^{17}\end{array}$ & $123 *$ & $\begin{array}{l}\text { Estudio observacional } \\
\text { retrospectivo }\end{array}$ & $\begin{array}{l}\text { - Los pacientes vistos en consulta ERCA son cada } \\
\text { vez menos y han aumentado los que entran } \\
\text { urgentemente. } \\
\text { - Los pacientes en DP tienen una edad y un índice } \\
\text { de comorbilidad menor que los de HD. } \\
\text { - El inicio programado fomenta la utilización de DP. }\end{array}$ \\
\hline $\begin{array}{l}\text { Gago MC, Martínez S, } \\
\text { Sesmero C et al. } 2007 . \\
\text { España }^{18}\end{array}$ & $198^{*}$ & $\begin{array}{l}\text { Estudio observacional } \\
\text { prospectivo }\end{array}$ & $\begin{array}{l}\text { - Fundamental la remisión temprana a la consulta } \\
\text { de ERCA. } \\
\text { - La enfermera dentro del equipo multidisciplinar } \\
\text { mejora la adhesión al tratamiento permitiendo la } \\
\text { participación activa en la elección del TRS. }\end{array}$ \\
\hline $\begin{array}{l}\text { Lacson E, Wang W, De Vries } \\
\text { C et al. } 2011 . \text { EEUU }\end{array}$ & $20057^{*}$ & $\begin{array}{l}\text { Estudio observacional } \\
\text { multicéntrico }\end{array}$ & $\begin{array}{l}\text { - Asistir a una sesión informativa en prediálisis se } \\
\text { asoció con la selección más frecuente de diálisis } \\
\text { domiciliaria. } \\
\text { - La disminución de DP en EEUU se ha } \\
\text { producido a pesar de que los datos muestran, } \\
\text { mayor satisfacción y reducción del coste global. } \\
\text { Consecuencia de falta de formación, disponibilidad } \\
\text { de muchos centros de HD, falta de incentivos, y } \\
\text { educación prediálisis inadecuada. }\end{array}$ \\
\hline $\begin{array}{l}\text { WuW, Wang SY, Hsu KH, } \\
\text { et al. 2009. Taiwan }{ }^{20}\end{array}$ & $573^{*}$ & Estudio de cohortes & $\begin{array}{l}\text { - El tiempo trascurrido en educación prediálisis } \\
\text { es imprescindible, mejora la calidad de vida } \\
\text { y aumenta el número de pacientes en diálisis } \\
\text { domiciliaria. } \\
\text { - Factores estructurales plantean problemas en la } \\
\text { libre elección de TRS. }\end{array}$ \\
\hline
\end{tabular}




\section{[ Carmen Caro Domínguez, et al ]}

Influencia de la consulta de enfermedad renal crónica avanzada en la elección de modalidad de terapia renal sustitutiva

\begin{tabular}{|c|c|c|c|}
\hline Autor, año, país & Muestra & Diseño del estudio & Resultados \\
\hline $\begin{array}{l}\text { Curtis BM, Ravani P et al. } \\
\text { 2005. Canadá21 }\end{array}$ & $288^{*}$ & Estudio de casos-controles & $\begin{array}{l}\text { - Los pacientes con educación multidisciplinar } \\
\text { presentan mayor supervivencia, más jóvenes, } \\
\text { eligen diálisis domiciliaria y son referidos de } \\
\text { forma temprana. }\end{array}$ \\
\hline $\begin{array}{l}\text { Marrón B, Ortiz A, de } \\
\text { Sequera P. 2006. España }{ }^{22}\end{array}$ & $1503^{*}$ & $\begin{array}{l}\text { Estudio multicéntrico } \\
\text { retrospectivo }\end{array}$ & $\begin{array}{l}\text { - El comienzo programado se asoció con un mejor } \\
\text { estado y DP más prevalente, edad joven,.. } \\
\text { - Las Unidades de ERCA son más propensas a dar } \\
\text { una atención óptima. }\end{array}$ \\
\hline $\begin{array}{l}\text { Braden J, Taub K, } \\
\text { Vanderstraeten C et al. } \\
\text { 2008. Canada }{ }^{23}\end{array}$ & $70^{*}$ & Estudio randomizado & $\begin{array}{l}\text { - Al informar en prediálisis más pacientes } \\
\text { comienzan diálisis domiciliaria. } \\
\text { - La diálisis domiciliaria supone un ahorro de } \\
\text { recursos y mayor eficiencia. } \\
\text { - La supervivencia y calidad de vida parece similar } \\
\text { para HD y DP. Los pacientes en HD domiciliaria } \\
\text { pueden tener una mayor calidad de vida que los } \\
\text { de HD en el hospital. } \\
\text { - Los pacientes con DP tenían más probabilidades } \\
\text { de recibir una información excelente. }\end{array}$ \\
\hline $\begin{array}{l}\text { Gómez AC, 0jeda MA, } \\
\text { Carcamo J. 2011. España }{ }^{24}\end{array}$ & $151^{*}$ & $\begin{array}{l}\text { Estudio observacional } \\
\text { transversal retrospectivo }\end{array}$ & $\begin{array}{l}\text { - Todos los pacientes en DP son informados en la } \\
\text { consulta. } \\
\text { - Los pacientes educados sobre TRS, eligen la DP } \\
\text { como primera opción en un } 32 \% \text { de los casos } \\
\text { y un } 8 \% \text { de los pacientes que conocen otras } \\
\text { alternativas cambian de tratamiento. } \\
\text { - Tener acceso vascular funcionante condiciona la } \\
\text { elección de tratamiento. }\end{array}$ \\
\hline $\begin{array}{l}\text { Rebollo A, et al. } 2014 . \\
\text { España }\end{array}$ & $90^{*}$ & $\begin{array}{l}\text { Estudio observacional } \\
\text { analítico }\end{array}$ & $\begin{array}{l}\text { - Pocos pacientes son seguidos en la consulta ERCA } \\
\text { - No existen diferencias significativas en la calidad } \\
\text { de vida entre ambos grupos. } \\
\text { - El seguimiento de los pacientes permite el } \\
\text { cumplimiento de la ley. }\end{array}$ \\
\hline $\begin{array}{l}\text { Mendelssohn D, Mujais S, } \\
\text { Soroka S. 2009. USA }{ }^{26}\end{array}$ & $1303^{*}$ & $\begin{array}{l}\text { Estudio de cohorte } \\
\text { prospectiva }\end{array}$ & $\begin{array}{l}\text { - La mayoría de los pacientes pueden ser adecuados } \\
\text { para la DP. } \\
\text { - La educación sobre TRS, puede aumentar la } \\
\text { incidencia de DP. } \\
\text { - La edad es la principal causa de no elegibilidad } \\
\text { para DP. } \\
\text { - La educación sanitaria temprana favorece la } \\
\text { elección de DP. }\end{array}$ \\
\hline $\begin{array}{l}\text { Marrón B, Martínez JC, } \\
\text { Salgueira M. 2005. Países } \\
\text { Bajos }^{27}\end{array}$ & $621^{*}$ & $\begin{array}{l}\text { Estudio retrospectivo } \\
\text { multicéntrico }\end{array}$ & $\begin{array}{l}\text { - Inicio no planificado se asocia con la edad } \\
\text { avanzada, menos información sobre TRS y mayor } \\
\text { uso de HD. }\end{array}$ \\
\hline $\begin{array}{l}\text { Juergensen E, Wuerth D et al. } \\
\text { 2006. EE.UU } 28\end{array}$ & $146^{*}$ & $\begin{array}{l}\text { Estudio observacional } \\
\text { descriptivo }\end{array}$ & $\begin{array}{l}\text { - Los pacientes con DP están más satisfechos y su } \\
\text { tratamiento tiene menos impacto en sus vidas. }\end{array}$ \\
\hline $\begin{array}{l}\text { García S, Vinagre G, Arribas } \\
\text { P. 2012. España }{ }^{29}\end{array}$ & $46^{*}$ & $\begin{array}{l}\text { Estudio observacional } \\
\text { descriptivo }\end{array}$ & $\begin{array}{l}\text { - Hay un alto porcentaje de pacientes que } \\
\text { comienzan TRS de forma aguda. } \\
\text { - Elección de DP condicionada por factores } \\
\text { socioeconómicos y edad. }\end{array}$ \\
\hline
\end{tabular}




\section{[ Carmen Caro Domínguez, et al ]}

Influencia de la consulta de enfermedad renal crónica avanzada en la elección de modalidad de terapia renal sustitutiva

\begin{tabular}{|c|c|c|c|}
\hline Autor, año, país & Muestra & Diseño del estudio & Resultados \\
\hline Ortega F. 2010. España 30 & $20 * *$ & $\begin{array}{l}\text { Estudio de revisión } \\
\text { bibliográfica }\end{array}$ & $\begin{array}{l}\text { - Gran variabilidad en la utilización de DP } \\
\text { por la existencia de diferentes modelos de } \\
\text { sistemas sanitarios, expansión de los grandes } \\
\text { proveedores de diálisis, escasa formación de la } \\
\text { técnica, no planificación de TRS... }\end{array}$ \\
\hline $\begin{array}{l}\text { Khan SS, Xue JL, Kazmi } \\
\text { WH, et al. 2005. México }{ }^{31}\end{array}$ & 109321 * & $\begin{array}{l}\text { Estudio de cohorte } \\
\text { retrospectiva }\end{array}$ & $\begin{array}{l}\text { - Los } 6 \text { meses antes de iniciar diálisis son cruciales, } \\
\text { incluyendo el ajuste psicosocial, la colocación } \\
\text { de un acceso vascular y se asocia a una mejor } \\
\text { supervivencia a largo plazo. }\end{array}$ \\
\hline $\begin{array}{l}\text { Garrido MV, Sesmero C., } \\
\text { Portolés J.M.2006. } \\
\text { España }^{32}\end{array}$ & $70 *$ & Estudio retrospectivo & $\begin{array}{l}\text { - La familia influye en el cambio de opción de TRS. } \\
\text { - La principal preocupación de los pacientes en } \\
\text { DP es la peritonitis y la falta de espacio para } \\
\text { almacenar el material. } \\
\text { - Los pacientes tienen presión familiar negativa } \\
\text { hacia la DP. }\end{array}$ \\
\hline $\begin{array}{l}\text { Hernández ME, Ochando A } \\
\text { et al. 2007. España }{ }^{33}\end{array}$ & $77^{*}$ & $\begin{array}{l}\text { Estudio observacional } \\
\text { descriptivo transversal }\end{array}$ & $\begin{array}{l}\text { - No hay correlación entre la capacidad funcional y } \\
\text { la satisfacción de los pacientes. } \\
\text { - El grado de satisfacción en HD y DP está } \\
\text { determinado por variables diferentes. }\end{array}$ \\
\hline $\begin{array}{l}\text { Mendelssohn C, Jassal S, } \\
\text { et al. 2002. Reino Unido }\end{array}$ & $210^{*}$ & Estudio cuasi-experimental & $\begin{array}{l}\text { - Los nefrólogos de las Islas Británicas afirman que } \\
\text { la HD es usada en exceso, y la planificación de } \\
\text { diálisis da lugar a mayor proporción de pacientes } \\
\text { en diálisis domiciliaria. }\end{array}$ \\
\hline $\begin{array}{l}\text { Brown EA et al. } 2010 . \\
\text { Reino Unido }{ }^{35}\end{array}$ & $140^{*}$ & $\begin{array}{l}\text { Estudio multicéntrico } \\
\text { transversal }\end{array}$ & $\begin{array}{l}\text { - En pacientes de edad avanzada, la calidad de vida } \\
\text { fue mejor en los que eligen DP. } \\
\text { - Este estudio apoya fuertemente la oferta de DP a } \\
\text { todas las personas mayores adecuadas. }\end{array}$ \\
\hline $\begin{array}{l}\text { Viglino G, Neri L, Alloatti S. } \\
\text { 2007. Italia }\end{array}$ & $53066 *$ & $\begin{array}{l}\text { Estudio observacional } \\
\text { retrospectivo }\end{array}$ & $\begin{array}{l}\text { - Uso de DP mayor en zonas con menos centros } \\
\text { privados. } \\
\text { - Las tasas de reembolso hacen a la DP menos } \\
\text { favorable. } \\
\text { - La falta de familiaridad de los profesionales con la } \\
\text { DP influye en su elección. }\end{array}$ \\
\hline Arrieta J. 2010. España ${ }^{37}$ & $24 * *$ & $\begin{array}{l}\text { Estudio de revisión } \\
\text { bibliográfica }\end{array}$ & $\begin{array}{l}\text { - Los médicos deben conocer los costes de las } \\
\text { terapias para contribuir a la sostenibilidad del } \\
\text { sistema. } \\
\text { - HD y DP tienen una eficacia similar y costes } \\
\text { diferentes. }\end{array}$ \\
\hline
\end{tabular}

Muestra: *= número de pacientes; ** = número de artículos. 


\section{Descripción de las variables y discusión}

\section{Variables que tienen relación con la consulta de ERCA}

\section{Existencia de un programa de educación prediálisis multidisciplinar}

Un programa de educación prediálisis multidisciplinar en la consulta ERCA ha permitido concienciar mejor al paciente sobre su enfermedad y las opciones de tratamiento, ayudando a superar los temores a la diálisis y teniendo un impacto significativo en la frecuencia de la elección de las diferentes modalidades de diálisis ${ }^{13,14}$. De hecho, varios estudios han afirmado que el hecho de derivar al paciente a dicha consulta y ofrecerle todas las opciones de tratamiento dialítico que se adapten a sus necesidades y a sus preferencias, favorece la DP como técnica de inicio ${ }^{13,22,26}$.

Cuando se informa al paciente sobre las modalidades de diálisis disponibles de forma neutral, sin sesgos, siempre y cuando no existan contraindicaciones clínicas, el 50\% de ellos elige una técnica de diálisis domiciliaria, un porcentaje muy superior a lo que sucede realmente ${ }^{15,16}$. La tasa de incidencia de las técnicas domiciliarias puede llegar a ser hasta seis veces superior en los pacientes asistentes a un programa de educación prediálisis, mejorando el acceso a la DP y la HD domiciliaria ${ }^{17}$.

En un estudio español con 202 pacientes, se les preguntó a éstos si antes de iniciar el TRS conocían las distintas modalidades de tratamiento de diálisis, contestando el $82 \%$ haber sido informados sobre la HD y un $21 \%$ haberlo sido sobre la DP, con el resultado de que el $83,5 \%$ de los entrevistados iniciaron el tratamiento en HD y el $16,5 \%$ en $\mathrm{DP}^{14}$. Por tanto, parece evidente que la información de que dispone el paciente a la hora de elegir modalidad de TRS es fundamental en su elección, de tal manera que, si hay carencias en el conocimiento de una técnica se favorecerá la elección de la otra.

Ortega-Suárez ${ }^{30}$, en un estudio de revisión ha encontró "un mayor desconocimiento de los pacientes sobre la $D P^{\prime \prime}$ basándose en un estudio español llevado a cabo por Celadilla et $\mathrm{a}^{38}$ en el que se realizó la misma pregunta a una muestra de pacientes en diálisis, respondiendo más del $65 \%$ de los mismos que conocían bien 0 muy bien la HD frente al $20 \%$ que conocía la existencia de las técnicas domiciliarias.

Numerosos estudios han asociado la elección de la HD con el desconocimiento de los pacientes de la posibili- dad de escoger la opción domiciliaria ${ }^{18-24}$. Algunos autores destacan que el paternalismo médico está muy arraigado entre la población, lo que puede condicionar un sesgo informativo que predispone a elegir la HD. De hecho, algunos pacientes critican el tiempo dedicado a la información de las TRS por parte del nefrólogo, detectando que, la prescripción de HD se realiza de manera automática y no argumentada ${ }^{26,27}$. Al ser la HD la modalidad que más se utiliza, la DP queda infrautilizada como procedimiento en aquellos casos en los que podría resultar la más idónea.

Por todo ello, es muy importante la creación de procesos que garanticen la información adecuada a los pacientes en las consultas ERCA, como destacan numerosos autores $13,14,17-24,26,27$.

Otro aspecto relevante es el papel del profesional de enfermería en estas consultas. Se ha documentado que la consulta de ERCA de enfermería mejora los conocimientos deficientes previos del paciente, mejora la adhesión al tratamiento, aumenta el autocuidado y bienestar, aportándole tranquilidad y apoyo mediante videos explicativos, folletos y sesiones para afrontar la enfermedad, proporcionando una atención óptima, además de, contribuir a la participación activa del paciente en la toma de decisiones respecto a la elección de la TRS, con el consiguiente aumento de la elección de terapias domiciliarias ${ }^{17,18,25}$. A pesar de estas ventajas, este tipo de consultas no están extendidas a todos los pacientes con ERCA pues no existen en la mayoría de los servicios de nefrología, lo que puede ser debido a causas no clínicas ${ }^{15,18,22-24,26,27}$.

\section{Referencia oportuna. Diálisis programada}

Diferentes estudios han insistido en la importancia que tiene la incorporación precoz de los pacientes con ERCA a los programas de información prediálisis para ayudar en la elección de la técnica. Una información precoz de las diferentes técnicas dialíticas se ha mostrado eficaz para evitar la incorporación de forma urgente y la posibilidad de permitir al paciente elegir la técnica que mejor se adecue a su estilo de vida, siempre que no haya contraindicación médi$\mathrm{ca}^{13,15-18,20-22,24,26,29-31}$. Según un trabajo de Marrón B. et al. ${ }^{22}$ "La proporción de pacientes que eligen DP de manera programada es del $27 \%$ frente al $6 \%$ que la inician de modo urgente".

Una remisión temprana del paciente a la consulta ERCA ha permitido que los pacientes que desconfían de las técnicas domiciliarias cambien su visión acerca de la 
complejidad de las mismas al facilitar el contacto con pacientes que ya las han elegido32.

\section{Otras variables que influyen en la elección de TRS}

\section{Edad}

La edad se ha mostrado como un factor muy influyente en la elección de la modalidad de TRS. Los pacientes en DP presentan una edad media inferior a los pacientes en $\mathrm{HD}$, aspecto que tiene relación con la capacidad funcional del paciente que decide realizar el TRS de forma autónoma ${ }^{13-17,27,28,29,32-34}$.

Los pacientes más jóvenes son más autónomos y, a menudo trabajan, por lo que son más propensos a elegir la DP como la modalidad que mejor se adapta a sus necesidades, ya que, ofrece mayor flexibilidad y libertad personal. Hay que tener en cuenta que los pacientes de edad avanzada tienen más dificultad a la hora de comprender las diferencias entre las modalidades de diálisis, mientras que, aspecto que puede influir en la elección de la DP, pues es una opción que el paciente elige basándose en un adecuado conocimiento de la técnica. Es evidente que, la elección de DP en personas mayores suele estar influenciada negativamente por contraindicaciones psicosociales, y no tanto por factores clínicos.

Otro estudio, ha apoyado la elección de la DP en personas de edad avanzada ya que, evita la necesidad de transporte hacia y desde una Unidad; y los cambios bruscos en el estado hemodinámico que induce la HD, lo que determina a menudo la mala tolerancia de los pacientes de edad avanzada a la HD23. El hecho de que los responsables médicos piensen que la DP no es factible para este grupo de pacientes puede conducir a un sesgo a la hora de ofrecer la información de las técnicas de diálisis.

\section{Comorbilidad}

Al ser la DP una técnica elegida por pacientes más jóvenes, es lógico que éstos presenten menor comorbilidad que los pacientes en HD, dada la repercusión que la edad tiene en el índice de comorbilidad ${ }^{17,26,28}$, siendo la Diabetes Mellitus la que más condiciona la elección de la DP17. De hecho, a medida que aumenta la comorbilidad asociada en el paciente en DP, la transferencia del paciente de esta técnica a HD es muy alta, ya que no pueden hacerse responsables de su tratamiento, perciben que son una carga para su familia y necesitan cuidados del profesional sanitario ${ }^{26,32}$.

\section{Factores económicos}

También hay que resaltar que los factores económicos pueden influir en la elección de modalidad de TRS. EI número de pacientes en diálisis aumenta exponencialmente en el mundo y todos los sistemas sanitarios se han planteado cómo restringir este gasto, debido a que los TRS son tratamientos muy costosos ${ }^{15}$.

Varios estudios ${ }^{15,37}$ han sugerido que, al impulsar la creación de consultas específicas de ERCA, se puede conseguir una mayor sostenibilidad y eficiencia del sistema sanitario, ya que, previenen y frenan la progresión de la ERCA hacia la TRS $y$, al informar de todas las modalidades de tratamiento permite que el paciente pueda elegir las terapias domiciliarias, las cuáles, son más económicas, ya que necesitan menor presencia de personal sanitario y estancia hospitalaria.

De hecho, un estudio realizado en Reino Unido ${ }^{34}$ ha establecido que la contención de costes favorece la expansión de la DP, que es menos costosa y suele estar acompañada de un descenso en la disponibilidad de HD.

En este sentido, es posible que la crisis favorezca la reestructuración del tratamiento sustitutivo de la ERCA, incidiendo en disminuir la proliferación descontrolada de puestos de HD que influye negativamente en la elección de DP y provocan un uso más eficiente de los recursos promocionando ésta última técnica ${ }^{30,41}$.

\section{Factores sociales}

Varios estudios ${ }^{27,29,34}$ han analizado la relación entre la actividad laboral con la elección de TRS, observando que existía una asociación con esta variable, es decir, hay más hombres jubilados que eligen HD y más hombres activos que eligen DP. Ser empleado y la flexibilidad que aportan las terapias domiciliarias, ha promovido su elección por parte del sector de pacientes activos.

La DP exige un fuerte compromiso. Algunos autores han señalado que la presencia de limitaciones físicas en el paciente y el hecho de necesitar colaboración familiar hace que los familiares ejerzan una presión negativa a asumir ese compromiso, ya que, supone un cambio en sus vidas; simultáneamente, los pacientes se sienten una carga para ellos ${ }^{16,20}$.

Otros estudios, sin embargo, han destacado que los pacientes que eligen DP tienen menos problemas sociales y dependencia física, menor índice de analfabetismo, mayor capacidad de comprensión y un buen soporte fa- 
miliar, por lo que, éstas no son barreras que dificultan su elección ${ }^{23,32}$.

\section{Aspectos estructurales}

Son varios los autores, que han confirmado la gran variabilidad en las tasas de incidencia y prevalencia de las diferentes modalidades de TRS entre países y entre diversas áreas geográficas en un mismo país, argumentando que, esto solo puede ser debido a factores estructurales y de organización ${ }^{15,30,40,41}$.

Se ha señalado en la bibliografía, la influencia que ejerce el tipo de provisión de los servicios sanitarios en la distribución de las modalidades de diálisis entre países. Los países con provisión pública, como los países Escandinavos y Reino Unido, presentan una mayor prevalencia de DP que los países con provisión sanitaria privada, países centroeuropeos y EE.UU. ${ }^{26,30,36}$. Hay excepciones a esta regla como Canadá y España, ya que, teniendo un sistema nacional de salud, tienen una baja proporción de pacientes en DP, por lo que, otras causas deben estar influyendo en la elección de la TRS.

Al analizar el descenso de la DP, Ortega-Suárez ${ }^{30}$ ha afirmado que éste se ha producido simultáneamente a la expansión de grandes multinacionales y cambio de propiedad de las unidades de diálisis, basándose en el estudio de Mehrotra et $a{ }^{42}$. Por otro lado, Portolés y Remón ${ }^{39}$ en una editorial afirman que la estructura sanitaria favorece la utilización de HD ofreciendo una amplia disponibilidad de puestos y relegando el uso de la DP como segunda técnica una vez saturada la HD.

Varios estudios han subrayado la importancia de la cultura y motivación de los profesionales de la salud en la elección de las terapias domiciliarias por parte del paciente ${ }^{15,20,30,36}$, ya que, esta cultura profesional se puede traducir en una inadecuada información al paciente en la etapa prediálisis. Además, Ortega $F^{41}$ ha afirmado que "a menor contacto con la DP, menos se utiliza por los médicos más jóvenes en formación".

Por otro lado, muchos nefrólogos han hecho referencia al esfuerzo que supone iniciar diálisis con terapias domiciliarias, ya que, disponen de un tiempo limitado e insuficiente para el cuidado que necesitan los pacientes en estas terapias, afirmando que es más fácil iniciar el tratamiento con HD15,19,20,30. También hay que considerar la percepción de los profesionales y de los propios pacientes de que "cuanto más máquina mejor asistencia sanitaria" y "la alta y cara tecnología es considerada como un signo de riqueza y progreso", lo que actúa en detrimento de la DP ${ }^{30}$.

\section{Factores psicológicos}

En varios artículos ${ }^{13,28}$, se ha establecido que los pacientes más optimistas son más propensos a elegir DP porque esta técnica proporciona más independencia, menor impacto en el ocio y nivel de energía.

Por otro lado, Rebollo et $\mathrm{a}^{25}$ ha señalado que los pacientes atendidos en la consulta ERCA presentan mayores niveles de ansiedad que los no atendidos, ya que, toman más conciencia de la importancia de su patología. En un estudio realizado en pacientes en DP se ha destacado la existencia de un alto porcentaje de pacientes con síntomas depresivos al inicio de elección de la técnica; sin embargo, estos síntomas no se han relacionado con la elección de la DP, salvo que se hayan producido complicaciones o reagudizaciones durante el tratamiento con ésta, en cuyo caso se produciría el cambio de esta técnica a HD ${ }^{16}$.

Nivel de satisfacción con la técnica de diálisis que reciben. Los pacientes en DP presentaron un nivel de satisfacción mayor que los pacientes en HD, teniendo además esta técnica, menos impacto en sus vidas $^{28,30,33,35}$. Tal vez esto no sea tan sorprendente, dado que el tratamiento se realiza en casa y no en el centro sanitario, permitiendo aumentar la capacidad de autonomía de los pacientes en DP frente a HD; aunque, la DPA por ejemplo, se realiza todas las noches, mientras que la HD tres veces por semana pero en turnos diurnos $^{28}$.

La variable que más ha influido en la satisfacción de los pacientes en DP han sido la información y la relación con los profesionales sanitarios que controlan su tratamiento, aspecto que se relaciona con la responsabilidad que asumen al realizarse la técnica una vez son informados. Sin embargo, los pacientes que reciben HD valoran más los aspectos relacionados con el trato y la confianza que transmite el personal y el hecho de poder interaccionar con otros pacientes de diálisis ${ }^{28,35}$.

No se han encontrado diferencias significativas en la calidad de vida en función de que hayan sido atendidos 0 no en la consulta de ERCA ${ }^{25}$; 0, según hayan sido tratados con una modalidad de diálisis u otra $(D P / H D)^{23}$. Dentro de la HD, los pacientes tratados con HD domiciliaria pueden tener una mayor calidad de vida relacionada con su salud en comparación con los de HD en el centro ${ }^{23}$. 


\section{Consideraciones prácticas}

La existencia de la consulta de ERCA en los centros de diálisis es fundamental para la libre elección de TRS por parte del paciente, haciendo que éste participe de forma activa en elegir la modalidad que más se adapte a sus necesidades, contribuyendo a aumentar el grado de satisfacción con la técnica.

La labor del profesional de enfermería está totalmente justificada a la vista de los resultados de esta revisión, pues se ha podido comprobar que la información o educación sanitaria más influyente en el paciente a la hora de elegir modalidad de TRS es la que este recibe de enfermería.

\section{Conclusiones}

A la vista de esta revisión, la variable más influyente en la elección de TRS es la existencia de las consultas de ERCA multidisciplinar en los servicios de nefrología; no obstante, se pone de manifiesto que algunos factores estructurales limitan la existencia de estas consultas, y por tanto, la información y atención que éstas proporcionan.

En concreto, las variables que permiten la elección de las técnicas domiciliarias son la existencia de un programa prediálisis multidisciplinar, la referencia oportuna del paciente a la consulta de ERCA, ser joven, menor comorbilidad, disponer de apoyo familiar y/o tener una red de apoyo y la necesidad de contención de costes sanitarios.

No obstante, la elección de las terapias domiciliarias queda obstaculizada por factores estructurales como la provisión sanitaria privada, expansión de grandes multinacionales de HD y la falta de especialización del profesional en las técnicas domiciliarias.

Los pacientes en DP están más satisfechos con su tratamiento que los incluidos en HD, ya que, el tratamiento tiene menos impacto en sus estilos de vida.

Recibido: 28 octubre 2016

Revisado: 5 noviembre 2016

Modificado: 20 noviembre 2016

Aceptado: 7 diciembre 2016

\section{Bibliografía}

1. Martínez-Castelao A. Documento de consenso para la detección y manejo de la enfermedad renal crónica. Atención primaria. 2014; 46(9):501/19. doi: 10.1016/j.aprim.2014.09.002.

2. Improving Global Outcomes (KDIGO) CKD Work Group. KDIGO Clinical Practice Guideline for the Evaluation and Management of Chronic Kidney Disease. Kidney Int. 2013; (Suppl. 3): 19-62. doi:10.1038/kisup.2012.64.

3. Orte L, Barril G. Unidad de enfermedad renal crónica avanzada (ERCA). Concepto de una unidad multidisciplinaria. Objetivos de la consulta de ERCA.Nefrología.2008;(Supl. 3):49-52.

4. Otero A, De Francisco A, Gayoso P, García F. On behalf of the EPIRCE Study Group. Prevalence of chronic renal disease in Spain: Results of the EPIRCE study. Nefrología. 2010; 30(1):78-86. doi: 10.3265/Nefrologia.pre2009.Dic.5732.

5. Arrieta J.Análisis coste-efectividad del tratamiento sustitutivo renal (hemodiálisis, diálisis peritoneal y trasplante) en España. La diálisis peritoneal en la planificación integral del tratamiento sustitutivo renal. 2010; 10 (5): 39-50.

6. Sánchez JA. Introducción a la Enfermedad Renal Crónica. En La Diálisis peritoneal en la planificación integral del tratamiento sustitutivo renal. Grupo de apoyo al Desarrollo de la Diálisis Peritoneal en España (GADPE). Madrid. España; 2010: 7-15.

7. Tejedor A, Sanz F, Pérez de Lucas N, López R, García Y, López JM, et al. Atención domiciliaria al paciente urémico terminal no susceptible de diálisis. Nefrología. 2006; 26(3): 66-81.

8. Bonilla FJ. Educación sanitaria al paciente con enfermedad renal crónica avanzada iexiste evidencia de su utilidad? Rev Soc Esp Enferm Nefro. [revista en Internet] 2014: abril-junio; [acceso 25 de enero de 2016] 17(2): 120-31. Disponible en: www.scielo.isciii.es/pdf/enefro/ v17n2/06_revision.pdf.

9. Gutiérrez JM, Ibars P, Pitarch G. Evaluación de los conocimientos adquiridos en la consulta prediálisis. Libro de comunicaciones del XXVII Congreso Nacional de la SEDEN. 2002: 180-5. 
10. Ley 41/2002, de 14 de noviembre, básica reguladora de la autonomía del paciente y de derechos y obligaciones en materia de información y documentación clínica. (Boletín Oficial del Estado, número 274, de 15-11-02).

11. Barril G, Sanz P, Ruperto M, Bardón E, Ruiz $P$, Selgas R. ¿Son necesarias las consultas específicas prediálisis? Objetivo: Cuidado integral del paciente con Enfermedad Renal Crónica (ERC). Nefrología. 2006; 26 (3): 33-41.

12. Górriz JL, Pallardó LM. Remisión precoz del paciente a nefrología: utopía o realidad. Nefrología. 2005; 25 (5): 16-21.

13. Ribitsch W, Haditsch B, Otto R, Schilcher $G$, Quehenberger $F$, Johannes $M$, Roob MJ, Rosenkranz AR. Effects of a pre-dialysis patient education program on the relative frequencies of dialysis modalities. Peritoneal Dialysis International. 2012; (33): 367-71. doi: 10.3747/ pdi.2011.00255.

14. Pastor JL, Julián JC. Claves del proceso de información y elección de modalidad de diálisis en pacientes con insuficiencia renal crónica. Nefrología. 2010; 1(1): 15-20.

15. Goovaerts T, Jadoul M, Goffin E. Influence of a Pre-Dialysis Education Programme (PDEP) on the mode of renal replacement therapy. Nephrol Dial Transplant. 2005; 20: 1842-47. doi:10.1093/ ndt/gfh905.

16. Ponz E, Martínez JC, Marquina D, Blasco C, Grau C, Mañé N, García N. Análisis de la influencia de los factores psicológicos en la elección de diálisis peritoneal. Nefrología. 2010;30(2):195-201.

17. Álvarez R, Velasco S. La consulta de prediálisis de enfermería: logros y oportunidades de mejora. Rev Soc Enferm Nefrol. 2007; 10 (3): 166-71.

18. Gago MC, Martínez $S$, Sesmero $C$, Andrés MM, Velayo $P$, Hernández $E$, et al. Influencia de la consulta prediálisis en los pacientes con enfermedad renal crónica avanzada. Enferm Nefrol. 2007; 1 (32): 76-80.

19. Lacson E, Wang W, De Vries C, Leste K, Hakim RM, Lazarus $M$, et al. Effects of a Nationwide
Predialysis Educational Program on Modality Choice, Vascular Access, and Patient Outcomes. Am J Kidney Dis. 2011; 58 (2): 235-42.

20. Wu W, Wang SY, Hsu KH, Lee $\mathrm{CH}$, Sun $\mathrm{CY}$, Tsai CJ, Wu MS. Multidisciplinary predialysis education decreases the incidence of dialysis and reduces mortality a controlled cohort study based on the NKF/DOQI guidelines. Nephrol Dial Transplant. 2009; 24: 2426-33. doi: 10.1093/ndt/gfp259.

21. Curtis BM, Ravani $P$, Maberti F, Kennett F, Taylor PA, Djurdjev 0 , et al. The short and long term impact of multidisciplinary clinics in addition to stan $\neg$ dard nephrology care on patient outcomes. Nephrol Dial Transplant. 2005; 20: 147-154.

22. Marron B, Ortiz A, Sequera $P$, Martín G, Arriba G, Lamas JM, Martínez JC, Arrieta J, Martínez F. Impact of end-stage renal disease care in planned dialysis start and type of renal replacement therapy a Spanish multicentre experience. Nephrol Dial Transplant. 2006; 21 (2): 51-5. doi:10.1093/ndt/ gfll91.

23. Manns BJ, Taub $K$, Vanderstraeten $C$, Jones $H$, Mills C, Visser M, Mclaughlin K. The impact of education on chronic kidney disease patients's plans to initiate dialysis with self-care dialysis: a randomized trial.Kidney International.2005; 68: 1777-83.

24. Gómez AC, Fernández D, 0jeda MA, Cabello $C$, Cárcamo J, Ramírez MA. Impacto de la educación sanitaria en la elección de la modalidad de diálisis. Enferm Nefrol. 2011; 36: 24-29.

25. Rebollo A, Morales JM, Pons ME. Influencia de la consulta de enfermería de enfermedad renal crónica avanzada en pacientes que inician tratamiento renal sustitutivo. Enferm Nefrol. 2014;17 (4): 243-250.

26. Mendelssohn DC, Mujais SK, Soroka SD et al. A prospective evaluation of renal replacement therapy modality Eligibility. Nephrol Dial Transplant. 2009; 24: 555-61. doi: 10.1093/ndt/ gfn484.

27. Marrón B, Martínez JC, Salgueira M, Barril G, Lamas JM, Martín M, et al. Analysis of patient flow into dialysis: role of education in choice of dialysis modality. Perit Dial Int. 2005; 25 (3): 56-9. 
28. Juergensen $E$, Wuerth $D$, Finkelstein $S H$, Juergensen $P$, Bekui A, Finkelstein F. Hemodialysis and Peritoneal Dialysis: Patients' Assessment of Their Satisfaction with Therapy and the Impact of the Therapy on Their Lives. Clin $\mathrm{J} \mathrm{Am}$ SocNephrol.2006; (1): 1191-96. doi: 10.2215/ CJN.01220406.

29. García $S$, Vinagre $G$, Arribas P. Influencia de factores epidemiológicos en la elección de la modalidad de tratamiento renal sustitutivo en la consulta de enfermería de enfermedad renal crónica avanzada. Rev Soc Esp Enferm Nefrol. 2012; 15 (4): 291-5.

30. Ortega F. Factores condicionantes en la elección del tratamiento sustitutivo renal. Dial Traspl. 2010;31(4):130-32.

31. Khan S,Xue JL,Kazmi WH, Tilbertson D, Obrador G, Pereira $B$, Collins AJ. Does predialysis nephrology care influence patient survival after initiation of dialysis? Kidney International. 2005; 67: 1038-46.

32. Garrido MV, Sesmero C, Portolés JM. Estudio sobre el cambio de elección de tratamiento renal sustitutivo en pacientes que han optado por diálisis peritoneal. Rev Soc Esp Enferm Nefrol. 2006; 9 (1): 7-11.

33. Hernández $M E$, Ochando $A$, Lorenzo $S$, Orbes $P$, López K. Factores determinantes de la satisfacción del paciente en tratamiento renal sustitutivo. Rev Soc Esp Enferm Nefrol. 2007; 10 (1): 6-13.

34. Mendelssohn C, Jassal S, et al.Attitudes of British Isles nephrologists towards dialysis modality selection: a questionnaire study. Nephrol Dial Transplant. 2002; 17: 474-77.

35. Brown EA, Johansson $L$, Farrington $K$, Gallagher H, Sensky T, Gordon F, Beckett N, Hickson M. Broadening Options for Long-term Dialysis in the Elderly (BOLDE): differences in quality of life on peritoneal dialysis compared to hemodialysis for older patients. Nephrol Dial Transplant. 2010; (25): 3755-63. doi: 10.1093/ndt/gfq212.
36. Viglino G, Neri L, Alloatti $S$, Cabiddu G, Cocchi R, Limido A, Marinangeli G, Russo R, Teatini U, Schena FP. Analysis of the factors conditioning the diffusion of peritoneal dialysis in Italy. Nephrol Dial Transplant. 2007; 22: 3601-5. doi:10.1093/ndt/ gfm416.

37. Arrieta J. Evaluación económica del tratamiento sustitutivo renal (hemodiálisis, diálisis peritoneal y trasplante) en España. Nefrología. 2010; 1(1):3747.

38. Celadilla 0 , Julve $M$, Vives $A$, De Miguel $M$, Arribas MJ, Cagigal D, et al. Evaluación de la información recibida por el paciente que inicia diálisis programada o procedente de trasplante. Libro de comunicaciones. XXXII Congreso SEDEN 07.

39. Viglino G, Neri L. Theory and reality in the selection of peritoneal dialysis. Peritoneal Dialysis International, 2008; 28 (5): 480-3.

40. Portolés J, Remón C. En busca de la eficiencia y sostenibilidad del tratamiento sustitutivo renal integrado. Nefrología. 2010; 1 (1): 2-7.

41. Ortega F. Influencia de los aspectos estructurales en el tratamiento sustitutivo renal. Nefrología. 2010; 1(1):21-5.

42. Mehrotra R, Khawar O, Duong U, Fried L, Norris K, Nissenson $A$, et al. Ownership patterns of dialysis units and peritoneal dialysis in the UnitedStates: utilization and outcomes. Am $\mathrm{J}$ Kidney Dis. 2009;54:289-98. 